\title{
Trastuzumab emtansine is the standard second-line treatment following horizontal blockade in first line of advanced HER-2 positive breast cancer: A plant that has still to grow
}

\author{
Liliana Montella $^{1 *}$, Salvatore Del Prete ${ }^{2}$ and Pasquale Bove ${ }^{1}$ \\ ${ }^{1}$ Naples 2 Local Health Unit, Italy \\ ${ }^{2}$ Naples 2 Local Health Unit, Oncology Operative Unit, Italy
}

Overexpression of human epidermal growth factor receptor 2 (HER2) occurs in 15\% to $25 \%$ of all breast cancers and is associated with poor prognosis.

From 2001 until now, treatment of HER-2 expressing breast cancer is radically changed. The cardinal role of HER-2 antigen [1] and the power of blocking HER-2 signalling by trastuzumab [2] have been increasingly recognized as the bricks on which a robust therapeutic strategy could be built. In fact, Cleopatra study in first-line [3], Emilia in second line [4] and Th3resa [5] in third line are only confirmative of this statement. The novel antibody pertuzumab, added to the trastuzumab in the triplet of Cleopatra study, significantly increases overall survival and progression-free survival as never before [3]. Trastuzumabado-emtansine (TDM-1) is an anti-HER2 antibody-drug conjugate (ADC). TDM-1 was pharmacologically developed on the solid axis of trastuzumab because the chemotherapeutic emtansine is too toxic to be used alone. This means that HER-2 pathway remains the way to arrive to tumor but the machine was empowered by a chemotherapeutic able to enhance the killing cell rate.

Before TDM-1 became commercially available, a patient with a metastatic HER-2 positive breast cancer was alive for more than 1 year with lapatinib and capecitabine [6]. Moreover, some consistent evidences favor trastuzumab beyond progression over lapatinib [7-9].

In second-line, after progression on trastuzumab, TDM-1 was able to significantly improve overall survival and progression-free survival by 5 and 3 months, respectively [4]. In 2017, a metastatic patient could survive above two years and half following treatment with TDM-1, about a doubling in overall survival as compares with treatment with lapatinib and capecitabine in 2008 [6].

Nowadays our challenge is the treatment of patients progressing after the gold standard first-line regimen of Cleopatra study and the answer is which therapy is better in second-line of HER-2 positive advanced/metastatic breast cancer: trastuzumab plus chemo- - or hormonal therapy in case of triple positive breast cancer- or trastuzumab emtansine? The phase II trial NCT01835236 with the aim to evaluate trastuzumab and pertuzumab followed by T-DM1 in metastatic breast cancer resulted active but not recruiting at the date of $15^{\text {th }}$ March, 2019 (https://clinicaltrials.gov/ct2/show/NCT01835236?term=NCT018 $35236 \&$ rank=1). However, this trial does not compare two different second-line strategies following trastuzumab plus pertuzumab, rather was aimed to weigh the value of a first-line approach without chemo. In fact, a double randomization was performed in first-line between double block only and double block plus chemo, and, thereafter, among those treated with chemotherapy, between paclitaxel and vinorelbine [10]. In this preliminary presentation, the Authors showed data on overall survival at 24 months as the primary end-point, which did not appear different between the two arms. In our opinion, the evaluation of survival at 24 months is little informative for two main reasons: it catches the early separation of patients with worse prognosis and outcome and it is only partially a mirror of the overall survival, which from pivotal Cleopatra study [3] is placed over 4 years.

Until now, no prospective study has been published reporting on treatment with trastuzumab emtansine following horizontal blockade with pertuzumab plus trastuzumab in first line. Therefore, have we enough elements coming from scientific background to prefer one treatment over another? Is TDM-1 better as second- or later-line choice? First of all, ASCO treatment guideline for HER-2 positive breast cancer recommends trastuzumab, pertuzumab, and taxane as firstline treatment and trastuzumab emtansine as second-line treatment [11]. In one small multiinstitutional series of 34 patients treated in second-line with trastuzumab emtansine, tumor response and PFS were worse in patients treated with first-line pertuzumab rather than after trastuzumab. In particular, overall response and progression free survival were both inferior [12]. Interestingly, the Authors reported the overall response rate to first-line anti-HER2 treatments, which was $51.7 \%$ with pertuzumab/trastuzumab and $63.9 \%$ in the trastuzumab group, respectively. This data means that patients treated with double blockade in the first line were probably different (more advanced? with brain/visceral metastases? hormone-receptor negative?) and fare worse than the other group. No detailed description of patients' characteristics was found. As comes from the PRAEGNANT Real-World Breast Cancer Registry [13] , Higher Eastern Cooperative Oncology Group (ECOG) scores, negative hormone receptor status, and visceral or brain

${ }^{*}$ Correspondence to: Liliana Montella, MD, Naples 2 Local Health Unit, 46 District, via Flavio Gioia, Acerra, Naples, Italy, E-mail: lilianamontella@libero.it

Received: February 12, 2019; Accepted: February 25, 2019; Published: February 27,2019 
Montella L (2019) Trastuzumab emtansine is the standard second-line treatment following horizontal blockade in first line of advanced HER-2 positive breast cancer: A plant that has still to grow

metastases were associated with more frequent use of the sequence pertuzumab-trastuzumab followed by T-DM1.

Another retrospective, observational study involving 23 cancer centres, and 250 patients treated with TDM- 1 reported a response rate of $41.6 \%$, median progression-free (mPFS) and median overall survival (mOS) of 6 and 20 months, respectively. Patients who received secondline T-DM1 had mPFS and mOS of 3 and 12 months $(\mathrm{p}=0.0001)$ if pertuzumab-pretreated, and 8 and 26 months if pertuzumab-naïve $(\mathrm{p}=0.06)$ [14]. TDM-1 performed better in pertuzumab-pretreated patients in third line and beyond. In fact, mPFS and mOS were 16 and 18 months in pertuzumab-pretreated $(\mathrm{p}=0.05)$ and 6 and 17 months in pertuzumab-naïve $(\mathrm{p}=0.30)$. In this study, T-DM1 showed greater efficacy when not administered immediately after pertuzumab-based treatment. This finding comes from 39 evaluable patients. Baseline characteristics between pertuzumab-naïve and pretreated patients were overall quite balanced and therefore no relevant difference was found. Many possible explanations can be suggested: a possible selection bias, i.e. choice of use TDM-1 in second-line in most aggressive disease, or biological reasons, i.e. temporary resistance to the anti-HER-2 therapy and/or the chemotherapeutic that has antimitotic interferences similar to docetaxel. The population evaluated in this study was mostly -above $70 \%$ - triple positive, i.e. HER-2 and hormone receptor positive, which is a kind of patients different from HER-2 positive-hormone receptor negative.

In a retrospective multicentric study published on JCO 2016, tumor response rate was $17.9 \%$ in a series of patients that received trastuzumab emtansine following trastuzumab and pertuzumab, which was lower than the $25.9 \%$ previously reported in a phase II study of a similar series [15].

Overall, data from selected patients treated with TDM-1 as exclusive second-line following trastuzumab and pertuzumab are scant.

During progression, a $7 \%-15 \%$ change in HER-2 status was described [16-18]. It is strongly recommended to use anti-HER-2 therapies beyond progression even in case of change in HER-2 status [19].

Does HER-2 blocking move during its natural history to a sort of "exhaustion" similarly to what happens for immune system when undergoes to continuous stimulation? I mean that acquired mechanisms of HER-2 resistance are behind the corner. From a biochemically point of view, T-DM1 and its metabolites need to accumulate in cell cytoplasm to reach a concentration that exceeds the threshold needed to evoke cell death [20]. Factors that affect the ability of the drug to reach a satisfactory intracellular level might be implicated in lack of clinical efficacy. Low tumor HER2 expression, poor internalization of the HER2-TDM1 complexes, defective intracellular and endosomal trafficking of the HER2-T-DM1 complex and defective lysosomal degradation of T-DM1 are all associated with low intracellular DM1 levels [21]. In addition, drug efflux pumps might be implicated in resistance to therapy and expression of multi-drug resistance transporters may play a role in T-DM1 resistance.

T-DM1 has robust single-agent activity in patients with heavily pretreated, HER2-positive MBC [22]. All clinicians should remember that, at any line of therapy, a rate of patients is lost. In two different studies concerning patients' series treated predominantly before 2010 , $3 \%$ and $26 \%$ of patients reached the goal of third-line treatment [23]. "Use the best drug first" seem to be the imperative in the modern oncology across various tumor algorithms.
We are reviewing records coming from four Institutions and concerning about one hundred females with advanced $\mathrm{BC}$ treated with TDM-1 with the aim to evaluate disease control and duration on therapy. Even with the previous limitations, we think that these investigations could be interesting because few data will come on this matter from prospective trials, given that TDM-1 as second-line is a recognized standard.

\section{References}

1. Slamon DJ, Clark GM, Wong SG, Levin WJ, Ullrich A, et al. (1987) Human breast cancer: correlation of relapse and survival with amplification of the HER-2/neu oncogene. Science 235: 177-182. [Crossref]

2. Slamon DJ, Leyland-Jones B, Shak S, Fuchs H, Paton V, et al. (2001) Use of Chemotherapy plus a Monoclonal Antibody against HER2 for Metastatic Breast Cancer That Overexpresses HER2. N Engl J Med 344: 783-792. [Crossref]

3. Swain SM, Baselga J, Kim SB, Ro J, Semiglazov V, et al. (2015) Pertuzumab, trastuzumab, and docetaxel in HER2-positive metastatic breast cancer. $N$ Engl J Med 372: 724-34. [Crossref]

4. Verma S, Miles D, Gianni L, Krop IE, Welslau M, et al. (2012) Trastuzumab emtansine for HER2-positive advanced breast cancer. $N$ Engl J Med 367: 1783-1791.

5. Krop IE, Kim SB, Martin AG, LoRusso PM, Ferrero JM, et al. (2017) Trastuzumab emtansine versus treatment of physician's choice in patients with previously treated HER2-positive metastatic breast cancer (TH3RESA): final overall survival results from a randomised open-label phase 3 trial. Lancet Oncol 18: 743-754. [Crossref]

6. Cameron D, Casey M, Press M, Lindquist D, Pienkowski T, et al. (2008) A phase III randomized comparison of lapatinib plus capecitabine versus capecitabine alone in women with advanced breast cancer that has progressed on trastuzumab: updated efficacy and biomarker analyses. Breast Cancer Res Treat 112: 533-543. [Crossref]

7. Hammerman A1, Greenberg-Dotan S1, Feldhamer I1, Bitterman H2, Yerushalmi R3 (2015) Second-Line Treatment of Her2-Positive Metastatic Breast Cancer: Trastuzumab beyond Progression or Lapatinib? A Population Based Cohort Study. PLoS One 10: e0138229. [Crossref]

8. Petrelli F, Barni S (2013) A pooled analysis of 2618 patients treated with trastuzumab beyond progression for advanced breast cancer. Clin Breast Cancer 13: 81-87. [Crossref]

9. Huober J, Baumann M, Rochlitz C, Aebi S, Güth U, et al. (2011) Trastuzumab treatment beyond progression in advanced breast cancer: patterns of care in six Swiss breast cancer centers. Oncology 81: 160-166. [Crossref]

10. Huober J, Weder P, Veyret C, Thürlimann B, Xyrafas A, et al. (2018) PERNETTA -A non comparative randomized open label phase II trial of pertuzumab (P) + trastuzumab(T) with or without chemotherapy both followed by T-DM1 in case of progression, in patients with HER2 positive metastatic breast cancer (SAKK 22/10 UNICANCER UC-0140/1207). Abstract presented at ESMO 2018.

11. Giordano SH, Temin S, Chandarlapaty S, Crews JR, Esteva FJ, et al. (2018) Systemic Therapy for Patients With Advanced Human Epidermal Growth Factor Receptor 2-Positive Breast Cancer: ASCO Clinical Practice Guideline Update. J Clin Oncol 36: 2736-2740. [Crossref]

12. Fabi A, Giannarelli D, Moscetti L, Santini D, Zambelli A, et al. (2017) Ado-trastuzumab emtansine (T-DM1) in HER2+ advanced breast cancer patients: does pretreatment with pertuzumab matter? Future Oncol 13: 2791-2797. [Crossref]

13. Lux MP, Nabieva N, Hartkopf AD, Huober J, Volz B, et al. (2019) Therapy Landscape in Patients with Metastatic HER2-Positive Breast Cancer: Data from the PRAEGNANT Real-World Breast Cancer Registry. Cancers (Basel) 11. [Crossref]

14. Vici P, Pizzuti L, Michelotti A, Sperduti I, Natoli C, et al. (2017) A retrospective multicentric observational study of trastuzumab emtansine in HER2 positive metastatic breast cancer: a real-world experience. Oncotarget 8: 56921-56931. [Crossref]

15. Dzimitrowicz H, Berger M, Vargo C, Hood A, Abdelghany O, et al. (2016) T-DM1 Activity in Metastatic Human Epidermal Growth Factor Receptor 2-Positive Breast Cancers That Received Prior Therapy With Trastuzumab and Pertuzumab. J Clin Oncol 34: 3511-3517. [Crossref]

16. Yang YF, Liao YY, Yang M, Peng NF, Xie SR, et al. (2014) Discordances in ER, PR and HER2 receptors between primary and recurrent/metastatic lesions and their impact on survival in breast cancer patients. Med Oncol 31: 214. [Crossref]

17. Sun L, Yu DH, Sun SY, Zhuo SC, Cao SS, et al. (2014) Expressions of ER, PR, HER-2, COX-2, and VEGF in primary and relapsed/metastatic breast cancers. Cell Biochem Biophys 68: 511-516. [Crossref] 
Montella L (2019) Trastuzumab emtansine is the standard second-line treatment following horizontal blockade in first line of advanced HER-2 positive breast cancer: A plant that has still to grow

18. Lindström LS, Karlsson E, Wilking UM, Johansson U, Hartman J, et al. (2012) Clinically used breast cancer markers such as estrogen receptor, progesterone receptor, and human epidermal growth factor receptor 2 are unstable throughout tumor progression. J Clin Oncol 30: 2601-2608. [Crossref]

19. Cardoso F, Senkus E, Costa A, Papadopoulos E, Aapro M, et al. (2018) 4th ESO-ESMO International Consensus Guidelines for Advanced Breast Cancer (ABC 4). Ann Oncol 29: 1634-1657. [Crossref]

20. Kovtun YV, Goldmacher VS (2007) Cell killing by antibody-drug conjugates. Cancer Lett 255: 232-240. [Crossref]
21. Barok M, Joensuu H, Isola J (2014) Trastuzumab emtansine: mechanisms of action and drug resistance. Breast Cancer Res 16: 209. [Crossref]

22. Burris HA III, Rugo HS, Vukelja SJ, et al. (2011) Phase II study of the antibody drug conjugate trastuzumab-DM1 for the treatment of human epidermal growth factor receptor 2 (HER2)-positive breast cancer after prior HER2-directed therapy. $J$ Clin Oncol 29: 398-405. [Crossref]

23. Manso L, Sanchez-Muñoz A, Calvo I, Izarzugaza Y, Plata J, et al. (2018) Late Administration of Trastuzumab Emtansine Might Lead to Loss of Chance for Better Outcome in Patients with HER2-Positive Metastatic Breast Cancer. Breast Care (Basel) 13: 277-283. [Crossref]

Copyright: (C2019 Montella L. This is an open-access article distributed under the terms of the Creative Commons Attribution License, which permits unrestricted use, distribution, and reproduction in any medium, provided the original author and source are credited. 